\title{
Anomalous Conductivity of Two-dimensional Dirac Electrons in Organic Conductor under Pressure
}

\author{
Yoshikazu Suzumura* $^{1}$ and Masao Ogata ${ }^{2}$ \\ ${ }^{1}$ Department of Physics, Nagoya University, Nagoya 464-8602, Japan \\ ${ }^{2}$ Department of Physics, University of Tokyo, Bunkyo, Tokyo 113-0033, Japan
}

\begin{abstract}
The electric conductivity of Dirac electrons in the organic conductor $\alpha$-(BEDT-TTF $)_{2} \mathrm{I}_{3}$ [BEDT-TTF $=$ bis(ethylenedithio)tetrathiafulvalene] under pressure has been examined using a two-dimensional tight-binding (TB) model with both impurity and electron-phonon (e-p) scatterings. We study an anomalous temperature dependence of the conductivity, which shows a crossover from $\sigma_{x}<\sigma_{y}$ at low temperatures [region (I)] to $\sigma_{x}>\sigma_{y}$ at high temperatures [region (II)]. $\sigma_{y}$ and $\sigma_{x}$ are diagonal conductivities parallel and perpendicular to a stacking axis of molecules, respectively. The effect of Dirac cone tilting is dominant in region (I), whereas the anisotropy of the velocity of the Dirac cone is dominant in region (II). Such behavior is further examined by calculating the deviation of principal axes due to the off-diagonal conductivity $\sigma_{x y}$ and a nearly constant conductivity at high temperatures due to the e-p scattering, which is the extension of the previous result of the simple two-band model [Phys. Rev. B 98,161205 (2018)]. The relevance to experiments of organic conductors is discussed.
\end{abstract}

\section{Introduction}

Since the discovery of two-dimensional massless Dirac fermions, ${ }^{1)}$ extensive studies have been performed on various materials. Among them, noticeable phenomena of Dirac electrons in molecular crystals ${ }^{2)}$ have been studied in the organic conductor $\alpha$-(BEDT-TTF $)_{2} \mathrm{I}_{3}{ }^{3)}$ [BEDT$\mathrm{TTF}=$ bis (ethylenedithio)tetrathiafulvalene]. After noting that the density of states (DOS) vanishes linearly at the Fermi energy, ${ }^{4)}$ the two-dimensional Dirac cone with a zero-gap state (ZGS) ${ }^{5}$ ) was found using a tight-binding (TB) model, where transfer energies are estimated from the extended Hückel method. ${ }^{6}$ ) The existence of such Dirac cone was verified by first-principles DFT calculation, ${ }^{7)}$ which has been used for studying further $\alpha$ $(\text { BEDT-TTF })_{2} \mathrm{I}_{3}$ under hydrostatic pressure. ${ }^{8)}$

There are common features among organic conductors with isostructure salts, ${ }^{9,10)} \alpha-\mathrm{D}_{2} \mathrm{I}_{3} \quad(\mathrm{D}=\mathrm{ET}$, $\mathrm{STF}$, and BETS), where ET = BEDT-TTF, STF = bis(ethylenedithio)diselenadithiafuluvalene), and BETS $=$ bis(ethylenedithio)tetraselenafulvalene. These salts display an energy band with a Dirac cone, ${ }^{5,11-13)}$ and the resistivity at high temperatures shows a nearly constant behavior, ${ }^{9,10,14-18)}$ whereas the conventional metal shows the linearly increasing one. Such unconventional behavior was also observed in Dirac electrons with nodal line semimetals of single-component molecular conductors $^{19-23)}$ Thus, it has been considered that the nearly constant behavior of resistivity at high temperatures is attributable to the intrinsic property of Dirac electrons.

Thus, the conductivity of Dirac electrons has been studied in simple models. A two-band model shows that the static conductivity at absolute zero temperature remains finite with a universal value, i.e., independent of the magnitude of impurity scattering owing to a quantum effect. ${ }^{24)}$ The conductivity increases with increasing doping concentration. The effect of Dirac cone tilting at absolute zero was studied previously. ${ }^{25)}$ It provides the anisotropic conductivity and the deviation of the current from the applied electric field. ${ }^{25)}$ At finite temperatures, on the other hand, the conductivity depends on the magnitude of the impurity scattering, $\Gamma$, which is proportional to the inverse of the life-time by the disorder. With increasing temperature $(T)$, the conductivity remains unchanged for $T \ll \Gamma$, whereas it increases for $\Gamma \ll T{ }^{26)}$ Noting that $\Gamma \sim 0.0003 \mathrm{eV}$ for organic conductors, ${ }^{2)}$ a monotonic increase in the conductivity at finite temperature $T>0.0005 \mathrm{eV}$ is expected. However the measured conductivity (or resistivity) on the above organic conductor shows an almost constant behavior at high temperatures. To comprehend such an exotic phenomenon, the acoustic phonon scatterings has been proposed as a possible mechanism, which was studied using a simple two-band model of the Dirac cone without tilting. ${ }^{27)}$ Although the scattering shows a reasonable suppression of the conductivity at high temperatures, it is unclear if such a model sufficiently explains the conductivity of the actual organic conductor, where the Dirac cone shows deviation from the linear spectrum and then the calculation must be performed beyond the model.

In this paper, we study the anomalous temperature dependence of the anisotropic conductivity of $\alpha$ (BEDT-TTF $)_{2} \mathrm{I}_{3}$ using the TB model ${ }^{8,28-30)}$ and acoustic phonons. Thus, the present paper is a natural extension of the previous study ${ }^{27}$ ) in which a simplified model was used without the details of the lattice structure of $\alpha$-(BEDT-TTF $)_{2} \mathrm{I}_{3}$. By using the TB model, we can automatically take into account the effects of tilting and anisotropy discussed at absolute zero ${ }^{25)}$ to explore the temperature dependence. It will be shown that the presence of acoustic phonons gives rise to conductivity being nearly constant at high temperatures. We will also

\footnotetext{
*E-mail: suzumura@s.phys.nagoya-u.ac.jp
} 


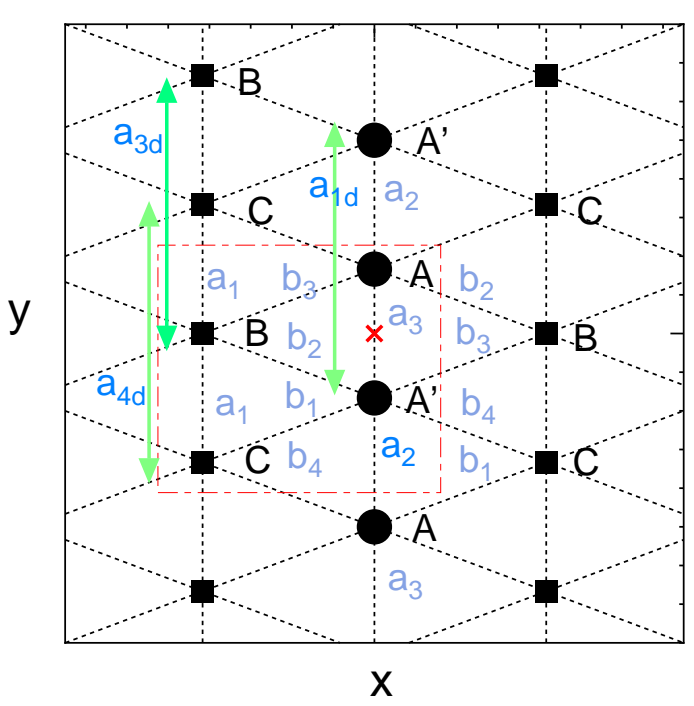

Fig. 1. (Color online) Crystal structure, where there are four molecules A, A', B, and C in the unit cell (dot-dashed line), which forms a square lattice. Transfer energies are shown by $a_{1}, \cdots, b_{4}$ for the nearest neighbor (NN) sites and $a_{1 d}, a_{3 d}$, and $a_{4 d}$ for the next-nearest neighbor (NNN) sites. The cross denotes an inversion center between $\mathrm{A}$ and $\mathrm{A}^{\prime}$.

demonstrate a crossover of the conductivity from a quantum regime at low temperatures to a classical regime at high temperatures by focusing on a competition between the tilted Dirac cone and the anisotropic velocity of the cone. We use the TB model with transfer energies of $\alpha$ $(\mathrm{BEDT}-\mathrm{TTF})_{2} \mathrm{I}_{3}$ under pressure, which are well known compared with those of other isostructural salts, BETS and STF. Since the tilted Dirac cone is also obtained for $\mathrm{BETS}^{12)}$ and STF, ${ }^{13)}$ it is expected that the present result provides a common feature for these salts, i.e., the nearly constant conductivity at high temperatures.

The paper is organized as follows. In Sect. 2, the model and formulation are given for both the uniaxial and hydrostatic pressures, where the latter case is examined by adding site potential. In Sect. 3, after examining the chemical potential and density of states (DOS), the conductivity is calculated for $\alpha$-(BEDT-TTF $)_{2} \mathrm{I}_{3}$ under both uniaxial and hydrostatic pressures. Section 4 is devoted to summary and discussion.

\section{Model and Formulation}

We consider a two-dimensional Dirac electron system per spin, which is given by

$$
H_{\text {total }}=H_{0}+H_{1}+H_{\mathrm{p}}+H_{\mathrm{e}-\mathrm{p}}+H_{i m p} .
$$

$H_{0}$ describes a TB model of the organic conductor $\alpha$ (BEDT-TTF $)_{2} \mathrm{I}_{3}$ consisting of four molecules per unit cell (Fig. 1), where $H_{1}$ describes a site potential obtained from a mean field of short-range repulsive interactions. ${ }^{8)}$ $H_{\mathrm{p}}$ and $H_{\mathrm{e}-\mathrm{p}}$ denote an acoustic phonon and an electronphonon $(\mathrm{e}-\mathrm{p})$ interaction, respectively. $H_{\mathrm{imp}}$ is the impurity potential. The terms $H_{0}+H_{\mathrm{p}}+H_{\mathrm{e}-\mathrm{p}}$ provide the Fröhlich Hamiltonian ${ }^{31)}$ applied to the present Dirac electron system. The unit of the energy is taken as eV.

\subsection{Energy band}

First, we derive the energy band for $H=H_{0}+H_{1}$ and the associated quantities. A TB model, $H_{0}$, is expressed as

$$
\begin{aligned}
H_{0} & =\sum_{i, j=1}^{N} \sum_{\alpha, \beta=1}^{4} t_{i, j ; \alpha, \beta} a_{i, \alpha}^{\dagger} a_{j, \beta} \\
& =\sum_{\boldsymbol{k}} \sum_{\alpha, \beta=1}^{4} t_{\alpha, \beta}(\boldsymbol{k}) a_{\alpha}^{\dagger}(\boldsymbol{k}) a_{\beta}(\boldsymbol{k}),
\end{aligned}
$$

where $a_{i, \alpha}^{\dagger}$ denotes a creation operator of an electron of molecule $\alpha\left[=\mathrm{A}(1), \mathrm{A}^{\prime}(2), \mathrm{B}(3)\right.$, and $\left.\mathrm{C}(4)\right]$ in the unit cell at the $i$-th lattice site. $N$ is the total number of square lattice sites and $t_{i, j ; \alpha, \beta}$ are the transfer energies for the nearest neighbor (NN) and nextnearest neighbor (NNN) sites, which are shown in Fig. 1. A Fourier transform for the operator $a_{j, \alpha}$ is given by $a_{j, \alpha}=1 / N^{1 / 2} \sum_{\boldsymbol{k}} a_{\alpha}(\boldsymbol{k}) \exp \left[i \boldsymbol{k} \cdot \boldsymbol{r}_{j}\right]$, where $\boldsymbol{k}=\left(k_{x}, k_{y}\right)$ and the lattice constant is taken as unity. The quantity $H_{1}$ corresponds to a site potential, $V_{\alpha}$, acting on the $\alpha$ site, where $V_{\mathrm{A}}=V_{\mathrm{A}^{\prime}}$ due to an inversion symmetry around the cross in Fig. 1. The Hamiltonian $H_{1}$ is obtained as (Appendix A)

$$
\begin{aligned}
H_{1} & =\sum_{\alpha}\left(V_{\alpha}-V_{\mathrm{A}}\right) \hat{n}_{\alpha} \\
& =\tilde{V}_{\mathrm{B}} \hat{n}_{\mathrm{B}}+\tilde{V}_{\mathrm{C}} \hat{n}_{\mathrm{C}},
\end{aligned}
$$

where $\tilde{V}_{\alpha}$ denotes a potential measured from that of the A site and $\hat{n}_{\alpha}=a_{\alpha}^{\dagger}(\boldsymbol{k}) a_{\beta}(\boldsymbol{k})$. From Eqs. (2) and (3), $H$ is written $\mathrm{as}^{8)}$

$$
H=\sum_{\boldsymbol{k}} \sum_{\alpha, \beta} a_{\alpha}^{\dagger}(\boldsymbol{k}) h_{\alpha, \beta} a_{\beta}(\boldsymbol{k}),
$$

with $h_{\alpha, \beta}$ being the matrix element (Appendix A) and is diagonalized as

$$
H=\sum_{\boldsymbol{k}} \sum_{\gamma} c_{\gamma}^{\dagger}(\boldsymbol{k}) E_{\gamma}(\boldsymbol{k}) c_{\gamma}(\boldsymbol{k})
$$

where $E_{1}(\boldsymbol{k})>E_{2}(\boldsymbol{k})>E_{3}(\boldsymbol{k})>E_{4}(\boldsymbol{k})$ and

$$
\begin{aligned}
\sum_{\beta} h_{\alpha, \beta}(\boldsymbol{k}) d_{\beta \gamma(\boldsymbol{k})} & =E_{\gamma}(\boldsymbol{k}) d_{\alpha \gamma}(\boldsymbol{k}), \\
c_{\gamma}(\boldsymbol{k}) & =\sum_{\alpha} d_{\alpha \gamma}(\boldsymbol{k}) a_{\alpha}(\boldsymbol{k}) .
\end{aligned}
$$

The Dirac point $\left(\boldsymbol{k}_{\mathrm{D}}\right)$ is calculated from

$$
E_{1}\left(\boldsymbol{k}_{\mathrm{D}}\right)=E_{2}\left(\boldsymbol{k}_{\mathrm{D}}\right)=\epsilon_{\mathrm{D}} .
$$

The ZGS is obtained when $\epsilon_{\mathrm{D}}$ becomes equal to the chemical potential at $T=0$.

From $E_{\gamma}$, the local density $n_{\alpha}$ including both spin $\uparrow$ and $\downarrow$ is calculated as

$$
\begin{aligned}
n_{\alpha} & =\frac{2}{N} \sum_{\boldsymbol{k}}\left\langle\hat{n}_{\alpha}(\boldsymbol{k})\right\rangle_{H} \\
& =\frac{2}{N} \sum_{\boldsymbol{k}} \sum_{\gamma} d_{\alpha \gamma}^{*}(\boldsymbol{k}) d_{\alpha \gamma}(\boldsymbol{k}) f\left(E_{\gamma}(\boldsymbol{k})-\mu\right),
\end{aligned}
$$

which is determined self-consistently. $n_{\mathrm{A}}=n_{\mathrm{A}^{\prime}}$ owing 
to transfer energies being symmetric with respect to the inversion center between A and $\mathrm{A}^{\prime}$ in Fig. 1. In Eq. (7), $f(\epsilon)=1 /(\exp [\epsilon / T]+1)$ with $T$ being temperature in the unit of $\mathrm{eV}$ and $k_{\mathrm{B}}=1$. The chemical potential $\mu$ is determined from the three-quarter-filled condition, which is given by

$$
\frac{1}{N} \sum_{\boldsymbol{k}} \sum_{\gamma} f\left(E_{\gamma}(\boldsymbol{k})-\mu\right)=\int_{-\infty}^{\infty} \mathrm{d} \omega D(\omega) f(\omega)=3
$$

where

$$
D(\omega)=\frac{1}{N} \sum_{\boldsymbol{k}} \sum_{\gamma} \delta\left(\omega-E_{\gamma}(\boldsymbol{k})\right) .
$$

$D(\omega)$ denotes DOS per spin and per unit cell, which satisfies $\int \mathrm{d} \omega D(\omega)=4$. Note that $n_{\mathrm{A}}+n_{\mathrm{A}^{\prime}}+n_{\mathrm{B}}+n_{\mathrm{C}}=6$ from Eq. (8). We use $\mu(T)$ at finite $T$ and $\mu=\mu(0)$ at $T=0$.

In Eq. (1), the third term denotes the harmonic phonon given by $H_{\mathrm{p}}=\sum_{\boldsymbol{q}} \omega_{\boldsymbol{q}} b_{\boldsymbol{q}}^{\dagger} b_{\boldsymbol{q}}$ with $\omega_{\boldsymbol{q}}=v_{s}|\boldsymbol{q}|$ and $\hbar=1$, whereas the fourth term is the e-p interaction expressed as $^{31)}$

$$
H_{\mathrm{e}-\mathrm{p}}=\sum_{\boldsymbol{k}, \gamma} \sum_{\boldsymbol{q}} \alpha_{\boldsymbol{q}} c_{\gamma}(\boldsymbol{k}+\boldsymbol{q})^{\dagger} c_{\gamma}(\boldsymbol{k}) \phi_{\boldsymbol{q}}
$$

with $\phi_{\boldsymbol{q}}=b_{\boldsymbol{q}}+b_{-\boldsymbol{q}}^{\dagger}$. We introduce a coupling constant $\lambda=\left|\alpha_{\boldsymbol{q}}\right|^{2} / \omega_{\boldsymbol{q}}$, which becomes independent of $|\boldsymbol{q}|$ for small $|\boldsymbol{q}|$. The $\mathrm{e}-\mathrm{p}$ scattering is considered within the same band (i.e., intraband) owing to the energy conservation with $v \gg v_{s}$, where $v \simeq 0.05^{8)}$ denotes the averaged velocity of the Dirac cone. The last term of Eq. (1), $H_{\text {imp }}$, denotes a normal impurity scattering, which is introduced to obtain the finite conductivity and to avoid the infinite conductivity in the presence of only the e-p interaction. $^{32)}$

\subsection{Conductivity}

By using the component of the wave function $d_{\alpha \gamma}$ in Eq. (5c), we calculate the conductivity per spin and per site $\operatorname{as}^{33)}$

$$
\begin{aligned}
\sigma_{\nu \nu^{\prime}}(T)= & \frac{e^{2}}{\pi \hbar N} \sum_{\boldsymbol{k}} \sum_{\gamma, \gamma^{\prime}} v_{\gamma \gamma^{\prime}}^{\nu}(\boldsymbol{k})^{*} v_{\gamma^{\prime} \gamma}^{\nu^{\prime}}(\boldsymbol{k}) \\
\times \quad & \int_{-\infty}^{\infty} d \epsilon\left(-\frac{\partial f(\epsilon)}{\partial \epsilon}\right) \\
& \frac{\Gamma_{\gamma}}{\left(\epsilon-\xi_{\boldsymbol{k} \gamma}\right)^{2}+\Gamma_{\gamma}^{2}} \times \frac{\Gamma_{\gamma^{\prime}}}{\left(\epsilon-\xi_{\boldsymbol{k} \gamma^{\prime}}\right)^{2}+\Gamma_{\gamma^{\prime}}^{2}}, \\
v_{\gamma \gamma^{\prime}}^{\nu}(\boldsymbol{k})= & \sum_{\alpha \beta} d_{\alpha \gamma}(\boldsymbol{k})^{*} \frac{\partial h_{\alpha \beta}}{\partial k_{\nu}} d_{\beta \gamma^{\prime}}(\boldsymbol{k})
\end{aligned}
$$

where $\nu=x$ and $y . h=2 \pi \hbar$ and $e$ denote Planck's constant and electric charge, respectively. The quantity $\Gamma_{\gamma}$ denotes the damping of the electron of the $\gamma$ band given by

$$
\Gamma_{\gamma}=\Gamma+\Gamma_{\mathrm{ph}}^{\gamma},
$$

where the first term comes from the impurity scattering and the second term corresponding to the phonon scattering is given by ${ }^{27)}$ (Appendix B)

$$
\begin{aligned}
\Gamma_{\mathrm{ph}}^{\gamma} & =C_{0} R \times T\left|\xi_{\gamma, \boldsymbol{k}}\right|, \\
R & =\frac{\lambda}{\lambda_{0}},
\end{aligned}
$$

with $C_{0}=6.25 \lambda_{0} /\left(2 \pi v^{2}\right)$. For $v \simeq 0.05$ and $\lambda_{0} / 2 \pi v=$ 0.1 , we obtain $C_{0} \simeq 12.5(\mathrm{eV})^{-1}$. $R$ denotes a normalized e- $\mathrm{p}$ coupling constant.

In the following, we denote $\sigma_{x}, \sigma_{y}$, and $\sigma_{x y}$ instead of $\sigma_{x x}(T), \sigma_{y y}(T)$, and $\sigma_{x y}(T)$ for simplicity. In terms of $\sigma_{x}, \sigma_{y}$, and $\sigma_{x y}$, the current $\left(j_{x}, j_{y}\right)$ obtained from a response to an external electric field $\left(E_{x}, E_{y}\right)$ is written as

$$
\left(\begin{array}{l}
j_{x} \\
j_{y}
\end{array}\right)=\left(\begin{array}{cc}
\sigma_{x} & \sigma_{x y} \\
\sigma_{x y} & \sigma_{y}
\end{array}\right)\left(\begin{array}{l}
E_{x} \\
E_{y}
\end{array}\right) .
$$

The principal axis of the Dirac cone has an angle $\phi$ measured from the $k_{y}$ axis, where $-\pi / 2<\phi<\pi / 2$. When we denote the current and electric field in this axis direction as $j_{x}^{\prime}$ and $E_{x}^{\prime}$, we obtain

$$
\left(\begin{array}{l}
j_{x}^{\prime} \\
j_{y}^{\prime}
\end{array}\right)=\left(\begin{array}{cc}
\sigma_{-} & 0 \\
0 & \sigma_{+}
\end{array}\right)\left(\begin{array}{l}
E_{x}^{\prime} \\
E_{y}^{\prime}
\end{array}\right)
$$

where

$$
\begin{aligned}
& \left(\begin{array}{l}
j_{x}^{\prime} \\
j_{y}^{\prime}
\end{array}\right)=\left(\begin{array}{cc}
\cos \phi & \sin \phi \\
-\sin \phi & \cos \phi
\end{array}\right)\left(\begin{array}{l}
j_{x} \\
j_{y}
\end{array}\right), \\
& \left(\begin{array}{l}
E_{x}^{\prime} \\
E_{y}^{\prime}
\end{array}\right)=\left(\begin{array}{cc}
\cos \phi & \sin \phi \\
-\sin \phi & \cos \phi
\end{array}\right)\left(\begin{array}{l}
E_{x} \\
E_{y}
\end{array}\right) .
\end{aligned}
$$

The relations between $\left(\sigma_{-}, \sigma_{+}, \phi\right)$ and $\left(\sigma_{x}, \sigma_{y}, \sigma_{x y}\right)$ are

$$
\begin{aligned}
\sigma_{x} & =\left(\cos ^{2} \phi\right) \sigma_{-}+\left(\sin ^{2} \phi\right) \sigma_{+}, \\
\sigma_{y} & =\left(\sin ^{2} \phi\right) \sigma_{-}+\left(\cos ^{2} \phi\right) \sigma_{+}, \\
\sigma_{x y} & =\sin \phi \cos \phi\left(\sigma_{-}-\sigma_{+}\right),
\end{aligned}
$$

or inversely,

$$
\begin{aligned}
\tan 2 \phi & =\frac{2 \sigma_{x y}}{\sigma_{x}-\sigma_{y}}, \\
\sigma_{-} & =\frac{1}{2}\left[\sigma_{x}+\sigma_{y}-\sqrt{\left(\sigma_{x}-\sigma_{y}\right)^{2}+4 \sigma_{x y}^{2}}\right], \\
\sigma_{+} & =\frac{1}{2}\left[\sigma_{x}+\sigma_{y}+\sqrt{\left(\sigma_{x}-\sigma_{y}\right)^{2}+4 \sigma_{x y}^{2}}\right] .
\end{aligned}
$$

Note that $\sigma_{x y}$ is not a Hall conductivity and $\sigma_{x y}=\sigma_{y x}$ holds as in Eq. (15) in the case of zero magnetic field. ${ }^{34)}$ $\sigma_{x y}$ is finite when $\sigma_{-} \neq \sigma_{+}$. The sign of $\phi$ is chosen such that $\phi<0$ for $\sigma_{x y}>0$ and $\phi>0$ for $\sigma_{x y}<0$, where $0<|\phi|<\pi / 4$ for $\sigma_{y}>\sigma_{x}$ and $\pi / 4<|\phi|<\pi / 2$ for $\sigma_{x}>\sigma_{y}$.

\section{Results}

We calculate the conductivity for the TB model with transfer energies shown in Fig. 1. The direction of molecular stacking is given by the $y$ axis and that perpendicular to the stacking is given by the $x$ axis. The NN transfer 
energies are examined for both uniaxial pressure and hydrostatic pressure, while those of NNN sites are added only for hydrostatic pressure. ${ }^{7)}$ In the following calculations, the conductivity is normalized by $e^{2} / \hbar$ and the energy is scaled by eV. First, we examine the case of uniaxial pressure $(P=6 \mathrm{kbar})$ without site potential and then the case of hydrostatic pressure with site potential to comprehend the similarity and dissimilarity.

\section{$3.1 \alpha-(B E D T-T T F){ }_{2} I_{3}$ under uniaxial pressure}

For the uniaxial pressure $P$ (kbar), we take only transfer energies with NN neighbor sites, $t=a_{1}, \cdots, b_{4}(\mathrm{eV})$, which are estimated by the extended Hückel method and interpolation, ${ }^{5,6)}$

$$
t(P)=t(0)\left(1+K_{t} P\right)
$$

where $t(0)$ is given by $a_{1}(0), \cdots, b_{4}(0)=-0.028,-0.048$, $0.020,0.123,0.140,0.062$, and 0.025 , and the corresponding $K_{t}$ is written as $0.089,0.167,-0.025,0,0.011$, and 0.032 , respectively. Since the ZGS is obtained for $P>3$ kbar, we choose $P=6 \mathrm{kbar}$ as a typical pressure.

Figure 2(a) shows two bands of $E_{1}(\boldsymbol{k})$ and $E_{2}(\boldsymbol{k})$, which touch at Dirac points $\pm \boldsymbol{k}_{\mathrm{D}}= \pm(0.57,0.30) \pi$ with an energy $\epsilon_{\mathrm{D}}=\mu=0.178$ corresponding to the threequarter-filled band. The ranges of the energy of the conduction and valence bands $E_{1}(\boldsymbol{k})$ and $E_{2}(\boldsymbol{k})$ are given by $0<E_{1}(\boldsymbol{k})-\epsilon_{\mathrm{D}}<0.19$ and $-0.11<E_{2}(\boldsymbol{k})-\epsilon_{\mathrm{D}}<0$, respectively. Such ZGS shows the relation $E_{2}(Y)<$ $\epsilon_{\mathrm{D}}<E_{1}(X)<E_{1}(M)$, where $\Gamma, \mathrm{X}, \mathrm{Y}$, and $\mathrm{M}$ are TRIMs given by $\Gamma=(0,0) \pi, X=(1,0) \pi, Y=(0,1) \pi$, and $M=(1,1) \pi$. Figure $2(\mathrm{~b})$ shows contour plots of $E_{1}(\boldsymbol{k})-E_{2}(\boldsymbol{k})$ as the function of $\delta \boldsymbol{k}=\boldsymbol{k}-\boldsymbol{k}_{\mathrm{D}}$ in a small region around $\boldsymbol{k}_{\mathrm{D}}$. The contour lines form almost isotropic circles, suggesting that the velocity of the Dirac cone is isotropic. Figure $2(\mathrm{c})$ shows $E_{1}(\boldsymbol{k})-\epsilon_{\mathrm{D}}$. The Dirac point is located at $\left(\delta k_{x}, \delta k_{y}\right)=(0,0)$. This contour suggests a tilted Dirac cone and shows a slight deviation from the ellipse. It is also found that the cone shows a slight rotation clockwise from the $k_{x}$ axis, which plays a crucial role in the transport property, as shown later. Figure $2(\mathrm{~d})$ shows $E_{2}(\boldsymbol{k})-\epsilon_{\mathrm{D}}$. The Dirac point is located at $(0,0)$. The contour of $E_{2}(\boldsymbol{k})-\epsilon_{\mathrm{D}}$ also shows a tilted Dirac cone and a slight deviation from the ellipse. We define a phase $\phi_{1}(<0)\left(\phi_{2}\right)$ as a tilting angle of $E_{1}(\boldsymbol{k})\left(E_{2}(\boldsymbol{k})\right)$ measured from the $k_{x}$ axis. Since $E_{1}(\boldsymbol{k})$ and $E_{2}(\boldsymbol{k})$ form a pair of Dirac cones, $\phi_{2}-\phi_{1}=\pi$ for $\boldsymbol{k}$ in the limit of the Dirac point. The deviation from the limiting value increases with increasing $\left|\delta \boldsymbol{k}_{\mathrm{D}}\right|$. The tilting parameter, which is a ratio of the tilting velocity to that of the Dirac cone, is given by $\eta \sim 0.8$. Figure $2(\mathrm{e})$ shows contour plots of $E_{1}(\boldsymbol{k})+E_{2}(\boldsymbol{k})-2 \epsilon_{\mathrm{D}}$. The bright line, $E_{1}(\boldsymbol{k})+E_{2}(\boldsymbol{k})-2 \epsilon_{\mathrm{D}}=0$, is perpendicular to the tilting direction of the cone.

Figure 3 shows the $T$ dependence of the chemical potential $\mu(T)$ for $P=6$ (solid line) and a hydrostatic pressure $P_{\text {hydro }}$ (dashed line) examined in the next subsection, where the corresponding DOS as a function of $\omega-\mu$ is shown in the inset. With increasing $T, \mu$ varies slowly, suggesting that the $T$ dependence of $\mu$ on $\sigma_{\nu}$ is negligibly small. In fact, the $T$ dependence of $\mu$ in Eq. (14a) may be
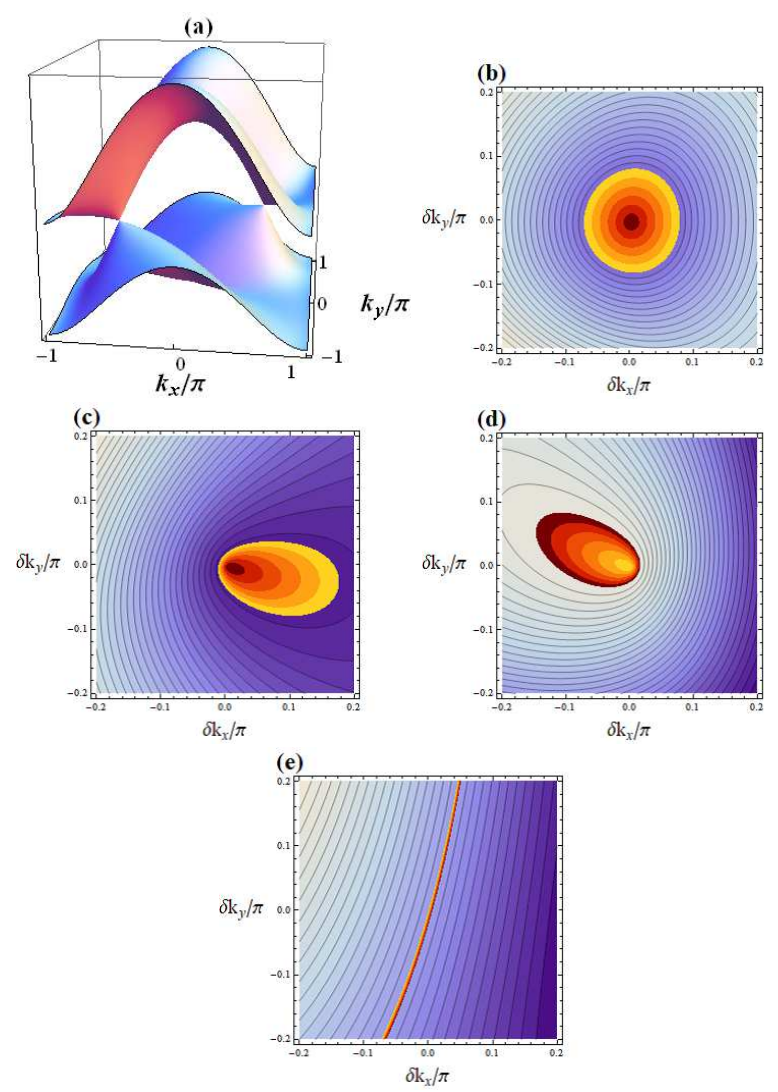

Fig. 2. (Color online) (a) Conduction and valence bands given by $E_{1}(\boldsymbol{k})$ (upper band) and $E_{2}(\boldsymbol{k})$ (lower band) at $P=6$ kbar. Two bands contact at the Dirac points $\pm \boldsymbol{k}_{\mathrm{D}}= \pm(0.57,0.30) \pi$ with an energy $\epsilon_{\mathrm{D}}=\mu=0.178$. (b) Contour plots of $E_{1}(\boldsymbol{k})-E_{2}(\boldsymbol{k})$ with the energy range $[0,0.106]$, where $\delta \boldsymbol{k}\left(=\boldsymbol{k}-\boldsymbol{k}_{\mathrm{D}}\right)$. The yellow line (outermost bright line) corresponds to $E_{1}(\boldsymbol{k})-E_{2}(\boldsymbol{k})=0.03$. (c) Contour plots of $E_{1}(\boldsymbol{k})-\epsilon_{\mathrm{D}}$ with the range [0, 0.096], where the Dirac point exists at $(0,0)$. The yellow line corresponds to $E_{1}(\boldsymbol{k})-\epsilon_{\mathrm{D}}=0.005$. (d) Contour plots of $E_{2}(\boldsymbol{k})-\epsilon_{\mathrm{D}}$ with the range $[-0.076,0]$. The Dirac point exists at $(0,0)$. The outermost bright line corresponds to $E_{2}(\boldsymbol{k})-\epsilon_{\mathrm{D}}=-0.005$. (e) Contour plots of $E_{1}(\boldsymbol{k})+E_{2}(\boldsymbol{k})-2 \epsilon_{\mathrm{D}}$ with the range [-0.062, 0.086]. The bright color line denotes $E_{1}(\boldsymbol{k})+E_{2}(\boldsymbol{k})-2 \epsilon_{\mathrm{D}}=0$.

ignored for $\sigma_{\nu}(T)$ at low temperatures. We verified that $\mu(T)$ can be replaced by $\mu(0)$ in $\xi_{\gamma, \boldsymbol{k}}$ for $0<T<0.015$ $(0<T<0.013)$, which is the range of temperatures of the following numerical calculation of $\sigma_{\nu}$ under the pressure of $P=6 \mathrm{kbar}$ ( $\left.P_{\text {hydro }}\right)$. The DOS close to the chemical potential shows a clear deviation from the linear dependence with respect to $\omega-\mu$, where $\mu$ is the chemical potential at $T=0$. Such asymmetry of DOS is ascribed to the nonlinear spectrum of the Dirac cone, where $E_{1}(\boldsymbol{k})\left(E_{2}(\boldsymbol{k})\right)$ is convex downward (upward) on the tangent plane at the Dirac point. The slight decrease in the chemical potential at low temperatures comes from such asymmetry. The increase in $\mu$ above the minimum occurs since the van Hove singularity at $E_{2}(Y)$ below the chemical potential has a large peak compared with that of $E_{1}(X)$.

Figure 4 shows the temperature dependence of conductivity of $\sigma_{\nu}(\nu=x, y, x y,+,-)$ without the e-p interaction, where $P=6$ and $\Gamma=0.0005$. Note that the solid line was already obtained in Ref. 33 but $\sigma_{x y}$ was absent 


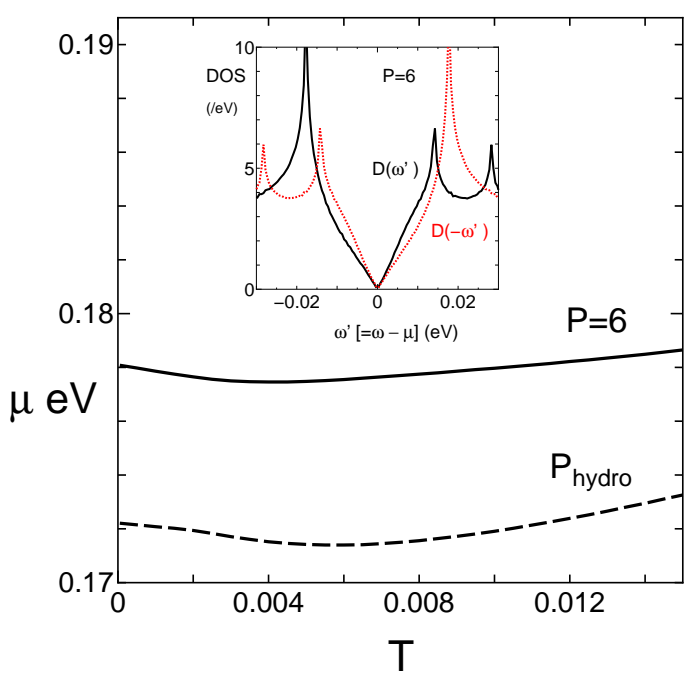

Fig. 3. (Color online) Chemical potential $(\mu)$ as a function of temperature $(T)$ for $P=6$ (solid line) and $P=P_{\text {hydro (dashed line). }}$ It is found that $\mu(T)$ for $P=6\left(P=P_{\text {hydro }}\right)$ takes a minimum at $T \sim 0.004(T \sim 0.006)$. The inset denotes the corresponding DOS as a function of $\omega-\mu=\omega^{\prime}$ for $P=6$, where $\mathrm{D}\left(-\omega^{\prime}\right)$ (red dotted line) is compared with $\mathrm{D}\left(\omega^{\prime}\right)$ showing the deviation from the linear dependence.

since the energy band with the Dirac cone was found later. ${ }^{5)}$ We discuss $\sigma_{\nu}(T)$ by dividing temperatures into two regions: (I) low temperatures with $\sigma_{y}$ being larger than $\sigma_{x}$ and (II) high temperatures with $\sigma_{x}$ being larger than $\sigma_{y}$.

First, we examine region (I), where the quantum region is found, leading to the finite conductivity even at $T=0$. In fact, the conductivity in the zero limit of $T$ is given by $\sigma_{x}(0) \simeq 0.06$ and $\sigma_{y}(0) \simeq 0.09$, which are compared with that of the universal value of $1 / 2 \pi^{2}=0.051$ obtained for the simple Dirac cone. The slightly larger value in the present case comes from the tilting of the Dirac cone. ${ }^{25)}$ An equality $\sigma_{y}>\sigma_{x}$ at low temperatures is understood as follows. Figure 2(b) shows a nearly isotropic velocity of the Dirac cone, whereas Figs. 2(c) and 2(d) present a large tilting of the Dirac cone almost along the $k_{x}$ direction. Our previous calculation of a tilted Dirac cone at $T=0$ shows that the conductivity becomes maximum for the direction perpendicular to a tilting axis. ${ }^{25)}$ This finding explains $\sigma_{y}>\sigma_{x}$ in the present case. Such inequality still holds at finite temperatures above $T>\Gamma$. As a reference, the conductivity of the isotropic Dirac cone without tilting, $\sigma_{\text {iso }}$, is shown by the dot-dashed line for $0<T<0.005$. With increasing $T, \sigma_{\text {iso }}$, which is slightly lower than $\sigma_{x}$ at $T=0$, becomes larger than $\sigma_{x}$. Thus, the effect of tilting is found in region (I), where $\sigma_{y}>\sigma_{\text {iso }}>\sigma_{x}$. By comparing the solid line $(\Gamma=0.0005)$ with the dotted line $(\Gamma=0.001)$, we found that the effect of tilting is reduced for large $\Gamma$.

Next, we examine region (II), where $\sigma_{x}>\sigma_{y}$ is found for $T>0.012$. In region (II) the effect of the tilting is neglected but the difference between $\sigma_{x}$ and $\sigma_{y}$ still exists owing to the anisotropy of the velocity of the cone. Since the DOS is averaged owing to a factor $(-\partial f(\epsilon) / \partial \epsilon)$ in Eq. (11) having a broad distribution with a width

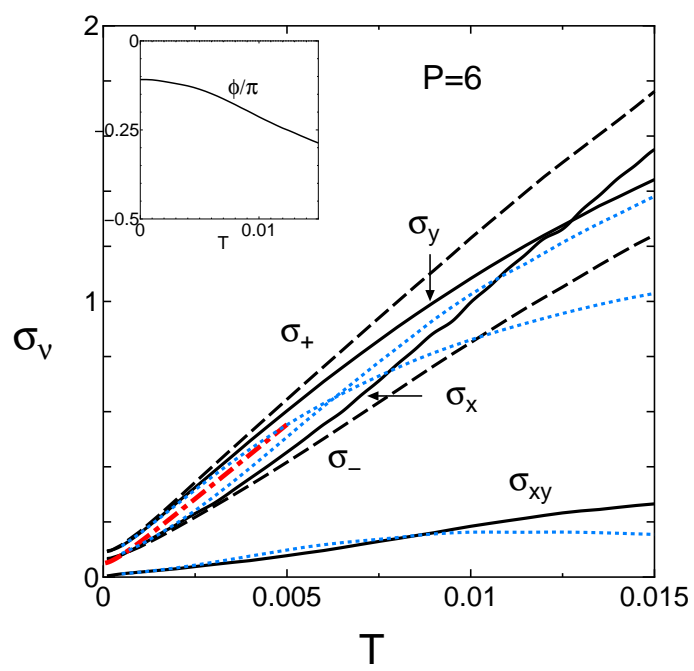

Fig. 4. (Color online) $T$ dependence of conductivity in the absence of the $\mathrm{e}-\mathrm{p}$ interaction at $P=6$ with fixed $\Gamma=0.0005$, where the solid lines denote $\sigma_{x}, \sigma_{y}$, and $\sigma_{x y}$ and the dashed lines denote $\sigma_{ \pm}$. The dotted lines correspond to $\Gamma=0.001$. As a reference, the conductivity of the isotropic Dirac cone without tilting, $\sigma_{\text {iso }}$, is shown by the (red) dot-dashed line for $0<T<0.005$. Principal values of $\sigma_{-}$and $\sigma_{+}$are given by Eqs. (18b) and (18c), respectively, whereas $\phi$ is given by Eq. (18a). The inset shows the phase $\phi$, which is an angle of the principal axis of $\sigma_{-}$measured from the $k_{x}$ axis.

$\sim T$, this resembles a classical conductivity with a finite chemical potential being $\sim T$. Thus, we find $\sigma_{x}>\sigma_{y}$, since the average velocity is larger for the $x$-direction than for the $y$-direction because the transfer energies are larger for the $x$-direction than for the $y$-direction. Such crossover occurs at lower $T$ for larger $\Gamma$. We also note that such crossover occurs at higher $T$ for $P=8$ (not shown here).

The dashed lines in Fig. 4 show the $T$ dependence of the principal value $\sigma_{ \pm}$. In the limit of $T=0$, the principal conductivity $\sigma_{+}$, which gives the maximum of the conductivity, becomes $\simeq \sigma_{y}$ and is perpendicular to the tilted Dirac cone. Note that this direction is almost parallel to the line of $E_{1}(\boldsymbol{k})+E_{2}(\boldsymbol{k})=0$ (see Fig. 2(e)). For $T \rightarrow 0$, we obtain $\sigma_{+}(0) \simeq 0.092$ and $\sigma_{-}(0) \simeq 0.0601$, which give $\sigma_{+}(0) / \sigma_{0} \simeq 1.82$ and $\sigma_{-}(0) / \sigma_{0} \simeq 1.32$ with $\sigma_{0}=1 /\left(2 \pi^{2}\right)$. These results are consistent with the analytical results of the tilted Dirac cone, where $\sigma_{+} / \sigma_{0}=$ 1.84 and $\sigma_{-} / \sigma_{0}=1.19$ for $\eta=084 .{ }^{25)}$ The behavior of $\sigma_{ \pm}$ being linear in $T$ resembles that of DOS around $\omega=\mu$ in the inset of Fig. 3, which is found for $|\omega-\mu|<0.015$. The inset shows the $T$ dependence of $\phi$, where $\phi<0$ owing to $\sigma_{x y}>0$ and $\sigma_{y}>\sigma_{x}$ at low temperatures, as seen from Eq. (18a). With increasing $T, \phi$ decreases and becomes smaller than $-\pi / 4$ at a temperature corresponding to $\sigma_{x}=\sigma_{y}$, where the axis close to $\sigma_{-}$changes from the $k_{x}$ axis to the $k_{y}$ axis. Thus, the principal axes rotate clockwise with an angle $\phi(<0)$ (see Eq. (16c)).

As shown in Fig. $4(P=6), \sigma_{\nu}$ increases monotonically as a function of $T$, which is different from the experiment showing nearly constant behavior at high temperatures. $^{2)}$ Such an exotic $T$ dependence of $\sigma_{\nu}$ is examined 


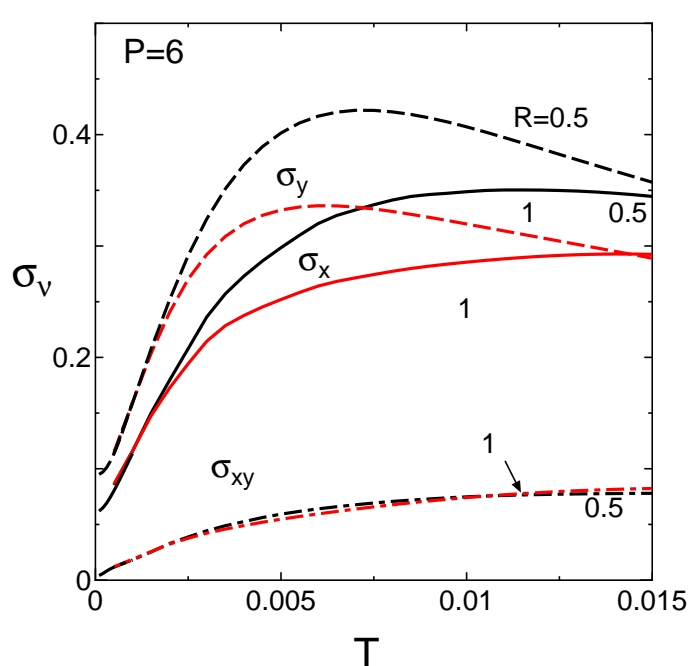

Fig. 5. (Color online) Conductivity $\sigma_{\nu}(\nu=x, y$, and $x y)$ in the presence of the e-p interaction with $P=6$ and $\Gamma=0.0005$. The solid, dashed, and dot-dashed lines denote $\sigma_{x}, \sigma_{y}$, and $\sigma_{x y}$, respectively, where $R$ denotes a normalized e-p coupling constant defined by the ratio $\lambda / \lambda_{0}$ (Eq. (14b)). $R=1$ corresponds to $\lambda=0.1$. At high $T, \sigma_{x}$ shows almost constant behavior and $\sigma_{y}$ shows a broad maximum.

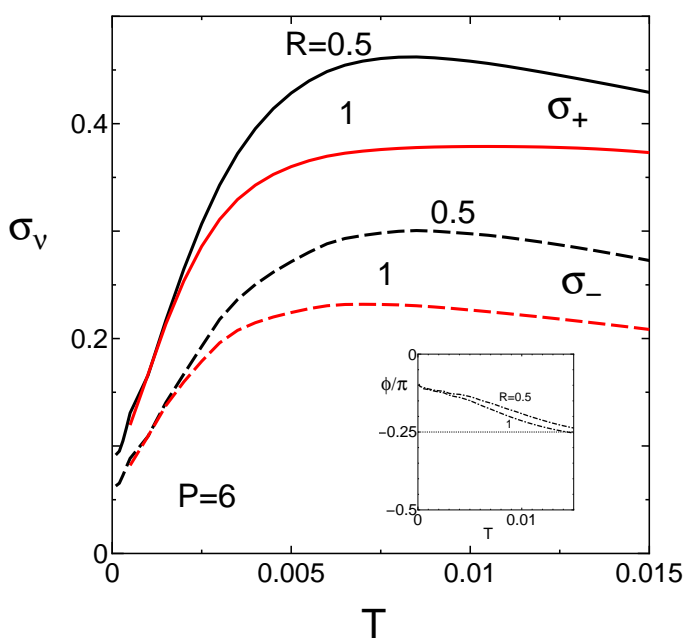

Fig. 6. (Color online) Conductivity $\sigma_{ \pm}$with $P=6$ and $\Gamma=$ 0.0005 in the presence of the $\mathrm{e}-\mathrm{p}$ interaction. The solid and dashed lines correspond to $\sigma_{+}$and $\sigma_{-}$, respectively, which show a broad maximum. The inset denotes the corresponding $\phi$.

next by taking account of the $\mathrm{e}-\mathrm{p}$ interaction, which is expected to reduce $\sigma_{\nu}$. By using Eq. (B.1b) and (14a), we calculate $\sigma_{\nu}$ of Eq. (11), where $\Gamma$ in the absence of the e-p interaction is replaced by $\Gamma_{\gamma}\left(=\Gamma+\Gamma_{\mathrm{ph}}^{\gamma}\right)$. Owing to the $T$ dependence of $\Gamma_{\mathrm{ph}}^{\gamma}, \Gamma$ is dominant at low $T$ whereas $\Gamma_{\mathrm{ph}}^{\gamma}$ is dominant at high $T$. Note that such crossover with increasing $T$ depends on $R$.

Figure 5 shows the $T$ dependence of $\sigma_{\nu}(\nu=x, y$, and $x y$ ) in the presence of the $\mathrm{e}-\mathrm{p}$ interaction with some choices of $R$. The effect of $R \neq 0$ appears when $\sigma_{\nu}$ deviates from the $T$-linear behavior. Compared with $\sigma_{\nu}$ with $R=0$ (Fig. 4), $\sigma_{\nu}$ is reduced noticeably. At temperatures around $T \sim 0.015, \sigma_{x}$ becomes nearly constant, whereas $\sigma_{y}$ takes a broad maximum at lower temperatures. The quantity $\sigma_{y}-\sigma_{x}$ decreases noticeably at high temperatures, suggesting that the effect of the tilting of the Dirac cone decreases with increasing temperature. The $R$ dependence on $\sigma_{x y}$ is small. The crossover temperature corresponding to $\sigma_{x}=\sigma_{y}$ decreases with increasing $R$, since the reduction of $\sigma_{y}$ is larger than that of $\sigma_{x}$. The nearly constant behavior of $\sigma_{x}$ is understood as follows. With increasing $T, \sigma_{\nu}$ without the e-p interaction $(R=0)$ increases linearly owing to the DOS obtained from the Dirac cone. Such a linear increase is suppressed for $R \neq 0$, since the noticeable effect of the acoustic phonon emerges at finite temperatures. In fact, the electron is scattered by both normal impurity $(\Gamma)$ and the e-p interaction $\left(\Gamma_{\mathrm{ph}}^{\gamma}\right)$, and the latter becomes dominant at high temperatures as seen from Eq. (14a). However, compared with a case of the conventional metal with a Fermi surface, the effect of the e-p scattering in the case of the Dirac cone close to the three-quarter-filled band is strongly reduced owing to a constraint by the energymomentum conservation. ${ }^{27)}$ This could possibly give rise to a nearly constant behavior or a broad maximum in $\sigma_{\nu}$, owing to a competition between the enhancement by DOS of the Dirac cone and the suppression by the e-p interaction.

Figure 6 shows the $T$ dependence of the principal values $\sigma_{ \pm}$corresponding to Fig. 5. Since $\sigma_{+}$and $\sigma_{-}$give the upper and lower bounds of the conductivity, these quantities are convenient for comprehending the results of experiments even when the precise relationship between the crystal axis and the direction of the applied electric field is unknown. In the inset, the angle between the $k_{x}$ axis and the tilting axis is shown, where $\phi<0$ for $\sigma_{x y}>0$ and $\sigma_{y}>\sigma_{x}$. The angle $|\phi|$ increases and exceeds $\pi / 4$ at a certain temperature with $\sigma_{x}=\sigma_{y}$, implying that the axis of $\sigma_{-}$becomes closer to the $k_{y}$ axis at high temperatures. Such a behavior can be understood on the basis of a simplified model, ${ }^{27)}$ where $\sigma_{+} \sim \sigma_{-} \sim \sigma$. Note that $\Gamma_{\mathrm{ph}}^{\gamma}$ is obtained in Eq. (14a) and $\sigma \simeq a_{\nu}^{\prime} 10^{3} T / \Gamma$ with $a_{\nu}^{\mathrm{ph}}=o(0.1)$ without $\mathrm{e}-\mathrm{p}$ interaction. By taking $\Gamma$ replaced by $\Gamma+\Gamma_{\mathrm{ph}}^{\gamma}$ and employing an idea $\left\langle\left|\xi_{\gamma, \boldsymbol{k}}\right|>\sim T\right.$ with $<>$ being an average value in the summation of Eq. (B.2), we obtain

$$
\sigma \simeq \frac{a_{\nu}^{\prime} 10^{3} T}{1+C_{0} R T^{2} / \Gamma},
$$

with $C_{0}=12.5$ and $\Gamma=0.0005$. From Eq. (20), it is found that a maximum of $\sigma$ as a function of $T$ is obtained by a competition of the DOS (the numerator) and the ep interaction (the denominator). Equation (20) suggests that $\sigma$ decreases with increasing $R$.

\section{2 $\alpha$-(BEDT-TTF $)_{2} I_{3}$ under hydrostatic pressures}

We examine Dirac electrons under hydrostatic pressure using the TB model with NN $\left(a_{1}, \ldots, b_{4}\right)$ and NNN $\left(a_{1 d}, \cdots, a_{4 d}\right)$ transfer energies given by ${ }^{7)} a_{1}=-0.0267$, $a_{2}=-0.0511, a_{3}=0.0323, b_{1}=0.1241, b_{2}=0.1296$, $b_{3}=0.0513, b_{4}=0.0152, a_{1 d}=0.0119, a_{3 d}=$ 0.0046 , and $a_{4 d}=0.0060$. The site potentials given by Eqs. $(\mathrm{A} \cdot 2 \mathrm{a})$ and $(\mathrm{A} \cdot 2 \mathrm{~b})^{8)}$ are estimated as $\tilde{V}_{B}=0.0511$, 
$\tilde{V}_{C}=0.0032$ (Appendix A).

Band energies $E_{j}(\boldsymbol{k})$ under hydrostatic pressure, which have Dirac cones similar to Fig. 2, are as follows. Figure 7 (a) shows the conduction and valence bands $(0<$ $E_{1}(\boldsymbol{k})-\epsilon_{\mathrm{D}}<0.15$ and $\left.-0.09<E_{2}(\boldsymbol{k})-\epsilon_{\mathrm{D}}<0\right)$ in the first Brillouin zone, which touch at the Dirac points $\pm \boldsymbol{k}_{\mathrm{D}}= \pm(0.69,0.44) \pi$ with $\epsilon_{\mathrm{D}}=0.172$. Compared with Fig. 2(a), the band width of both $E_{1}(\boldsymbol{k})$ and $E_{2}(\boldsymbol{k})$ is slightly large and the Dirac points move away from the $\Gamma$ point. In Fig. $7\left(\right.$ b), the energy difference between $E_{1}$ and $E_{2}$ is shown. The contour lines form ellipsoids, suggesting that the anisotropy of the velocity of the Dirac cone is larger than that of Fig. 2(b), e.g., an ellipse with the ratio of the major to minor axes being $\simeq 1.2$. Figure $7(\mathrm{c})$ shows that the tilting angle $\phi(>0)$ of $E_{1}(\boldsymbol{k})-\epsilon_{\mathrm{D}}$ measured from the $k_{x}$ axis has the sign opposite to that of Fig. 2(c). As shown later, such difference in the sign results in the difference in the current direction, i.e., the rotation with respect to the applied electric field of $E_{x}$ or $E_{y}$. Figure $7(\mathrm{~d})$ shows $E_{2}(\boldsymbol{k})-\epsilon_{\mathrm{D}}$ forming a pair of Dirac cones with that of Fig. 7(c), where the deviation of the tilting axis from the $k_{x}$ axis is opposite to that of Fig. 2(d). The tilting direction is understood from Fig. 7(e), where the energy of the Dirac point is given by $E_{1}\left(\boldsymbol{k}_{\mathrm{D}}\right)-\epsilon_{\mathrm{D}}=E_{2}\left(\boldsymbol{k}_{\mathrm{D}}\right)-\epsilon_{\mathrm{D}}=0$ and the tilting direction of the cone is almost perpendicular to the zero line $\left[E_{1}(\boldsymbol{k})+E_{2}(\boldsymbol{k})-2 \epsilon_{\mathrm{D}}=0\right]$. By comparing Figs. 2 with Figs. 7, we find the opposite rotation of the tilted cone.

Now we examine the electric conductivity. The parameters for $P_{\text {hydro }}$ are taken as $\Gamma=0.0005$ and $\tilde{V}_{B}=0.0511$, $\tilde{V}_{C}=0.0032$ for the site potentials, which are obtained from Eqs. (A.2a) and (A.2b). Figure 8 shows the conductivity $\sigma_{\nu}$ without the e-p interaction, where the solid line (dashed line) denotes $\sigma_{x}, \sigma_{y}$, and $\sigma_{x y}\left(\sigma_{ \pm}\right)$. Compared with the case of the uniaxial pressure (Fig. 4), the sign with $\sigma_{x y}<0$ is opposite, as also seen from a comparison of Figs. 7(e) and 2(e), showing that the angle of the zero line $E_{1}+E_{2}-2 \epsilon_{\mathrm{D}}=0$ from the $k_{y}$ axis is opposite. This difference originates from the transfer energies. A noticeable difference between hydrostatic pressure and uniaxial pressure exists in $\sigma_{\nu}$ at low temperatures, where $\sigma_{y}>\sigma_{x}$ in Fig. 4 and $\sigma_{y} \simeq \sigma_{x}$ in Fig. 8. The origin of $\sigma_{y} \simeq \sigma_{x}$ is understood as follows from Figs. 7(b)-7(d). Figure 7 (b) shows an anisotropy of the contour implying that the ratio of the maximum to minimum velocities of the Dirac cone is $\simeq 1.2$, which results in the ratio of the maximum and minimum conductivities being $\simeq 1.4{ }^{25}$ ) On the other hand, the tilted cone gives $\sigma_{y} / \sigma_{x} \simeq 1.4$ for the tilting parameter $\left.\eta \sim 0.9 .{ }^{25}\right)$ The competition of these two ingredients provides an almost isotropic conductivity at low temperatures. The conductivity at high temperatures is given by the classical region, where the effect of the tilting can be neglected and the larger transfer energies along the $x$ direction gives a larger velocity for the $k_{x}$ axis as seen from Fig. 7(b).

Figure 8 also shows the principal value $\sigma_{ \pm}$of the conductivity, where $\phi(>0)$ in the inset denotes an angle between the $\sigma_{-}$axis and the $k_{x}$ axis. With increasing $T$, the difference between $\sigma_{+}$and $\sigma_{-}$increases but is smaller than that shown in Fig. 4. This comes from a difference
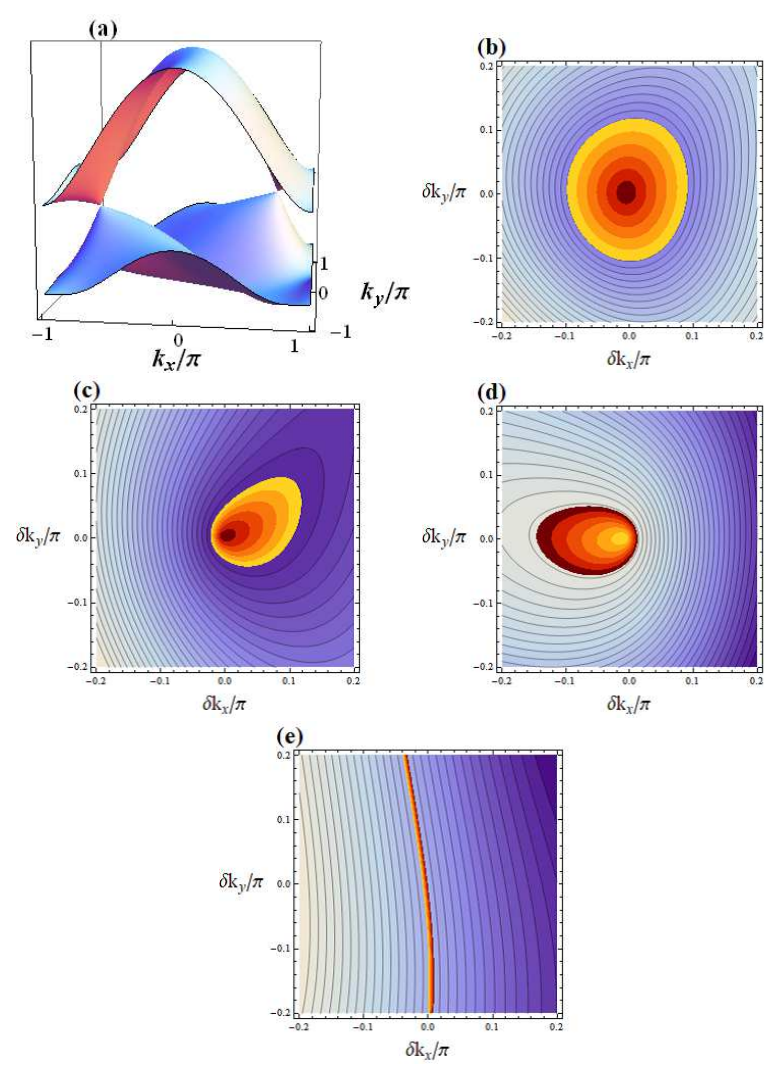

Fig. 7. (Color online) Energy bands of $E_{1}(\boldsymbol{k})$ and $E_{2}(\boldsymbol{k})$ under hydrostatic pressure, where the Dirac points are given by $\pm \boldsymbol{k}_{\mathrm{D}}=$ $\pm(0.69,0.44) \pi$ and $\mu=\epsilon_{\mathrm{D}}=0.172$. (a) Conduction and valence bands given by $E_{1}(\boldsymbol{k})$ (upper band) and $E_{2}(\boldsymbol{k})$ (lower band). (b) Contour plots of $E_{1}(\boldsymbol{k})-E_{2}(\boldsymbol{k})$ as a function of $\delta \boldsymbol{k}=\boldsymbol{k}-\boldsymbol{k}_{\mathrm{D}}$ with the energy range $[0,0.09]$. The yellow line (outermost bright line) corresponds to $E_{1}(\boldsymbol{k})-E_{2}(\boldsymbol{k})=0.03$. (c) Contour plots of $E_{1}(\boldsymbol{k})-\epsilon_{\mathrm{D}}$ as a function of $\delta \boldsymbol{k}$ with the range [0, 0.066]. The yellow line corresponds to $E_{1}(\boldsymbol{k})-\epsilon_{\mathrm{D}}=0.005$. (d) Contour plots of $E_{2}(\boldsymbol{k})-\epsilon_{\mathrm{D}}$ as a function of $\delta \boldsymbol{k}$ with the range [-0.056, 0]. The outermost colored region corresponds to $E_{1}(\boldsymbol{k})-\epsilon_{\mathrm{D}}=-0.005$. (e) Contour plots of $E_{1}(\boldsymbol{k})+E_{2}(\boldsymbol{k})-2 \epsilon_{\mathrm{D}}$ with the range [-0.045, $0.045]$. The bright color line denotes $E_{1}(\boldsymbol{k})+E_{2}(\boldsymbol{k})-2 \epsilon_{\mathrm{D}}=0$.

at low temperatures, where $\sigma_{y}-\sigma_{x}$ for the uniaxial pressure is larger than that for the hydrostatic pressure.

We discuss $\sigma_{\nu}$ with transfer energies of hydrostatic pressure but without site potentials, which is shown by the dot-dashed line in Fig. 8. In this case, $\sigma_{y} \simeq \sigma_{x}$ but $\sigma_{x y}>0$. This comes from the fact that the ellipse corresponding to Fig. 7(b) is slightly rotated anticlockwise (not shown here). Thus, it turns out that $\sigma_{y} \simeq \sigma_{x}$ comes from transfer energies of hydrostatic pressure and that $\sigma_{x y}>0$ is attributable to the absence of the interaction.

Now, we examine the effect of the e-p scattering on the $T$ dependence of conductivity with some choices of $R, \mathrm{Eq}$. (14b), where the effect of the site potential is included in the energy band. Figure 9 shows $\sigma_{\nu}$ with the fixed $R=0.5$ and 1 , in which sufficient suppression of $\sigma_{\nu}$ by $R$ is seen compared with that of Fig. $8(R=0)$. The crossover temperature, where $\sigma_{y}>\sigma_{x}$ at low temperatures and $\sigma_{x}>\sigma_{y}$ at high temperatures, decreases with increasing $R$. The increase toward a constant behavior at high $T$ is seen for $\sigma_{x}$, whereas a broad maximum is found for $\sigma_{y} . \sigma_{x y}$ as a function of $T$ takes a minimum followed 


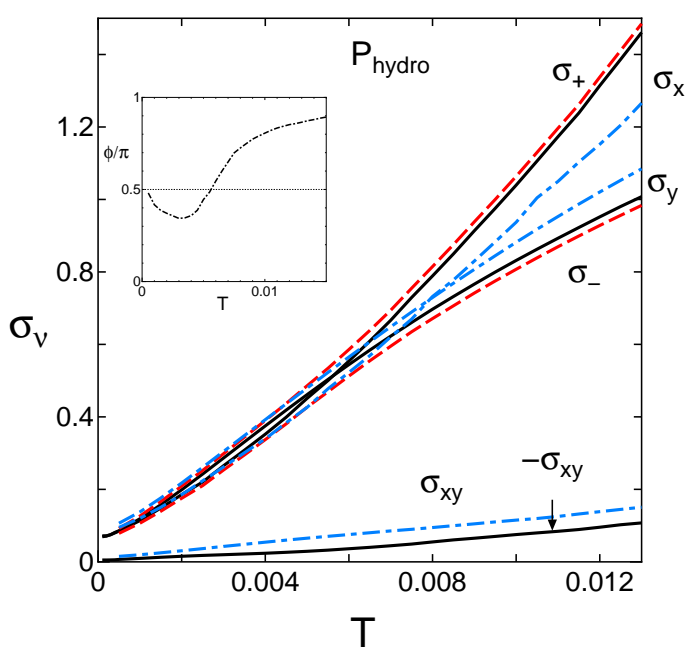

Fig. 8. (Color online) Conductivity $\sigma_{\nu}$ with $\nu=x, y$, and $x y$ (solid line), and $\nu= \pm$ (dashed line) under hydrostatic pressure. Note that $\sigma_{x y}<0$ and $\phi / \pi(>0)$ (inset) from Eq. (18a). The dotdashed line denotes $\sigma_{\nu}$ without the site potential, where $\mu(0)=$ 0.168

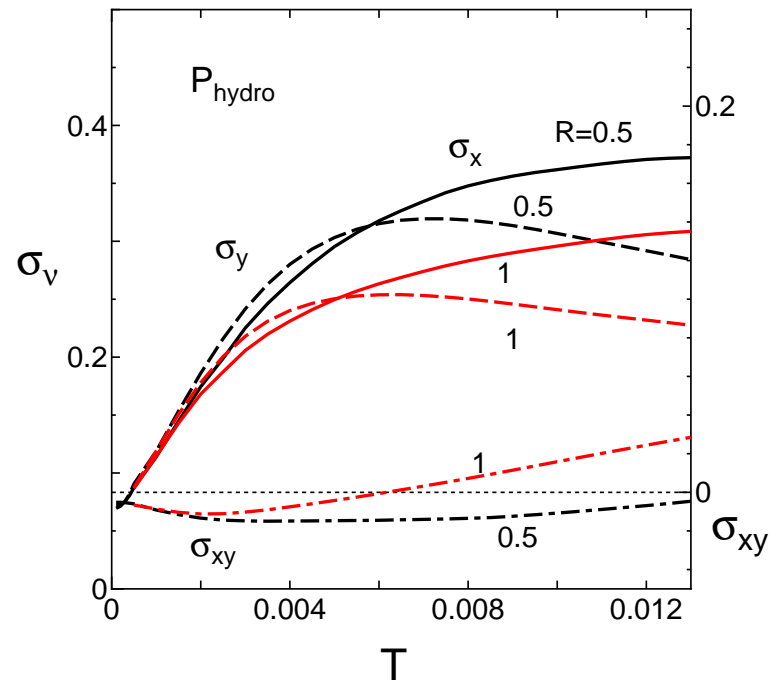

Fig. 9. (Color online) Conductivity $\sigma_{\nu}(\nu=x, y$, and $x y)$ under hydrostatic pressure in the presence of the $\mathrm{e}-\mathrm{p}$ interaction with choices of $R=0.5$ and 1 .

by the change of the sign for large R. Compared with Fig. 4, the crossover temperature of $\sigma_{y}=\sigma_{x}$ is lower for the hydrostatic pressure, where $\sigma_{y}$ is sufficiently reduced and $\sigma_{x}$ is less reduced.

\section{Summary and Discussion}

We calculated the $T$ dependence of conductivity $\sigma_{\nu}$ $(\nu=x$ and $y)$ of Dirac electrons in the organic conductor $\alpha$-(BEDT-TTF $)_{2} \mathrm{I}_{3}$ under both uniaxial and hydrostatic pressures to find that the anisotropic conductivity exhibits a crossover from region (I) including the quantum regime at low temperatures to region (II) showing the classical regime at high temperatures. The former with $\sigma_{y}>\sigma_{x}$ comes from the tilted Dirac cone, whereas the latter with $\sigma_{x}>\sigma_{y}$ originates from the anisotropy of the velocity of the cone, and a nearly constant conductivity comes from the phonon scattering on the Dirac cone. The presence of the off-diagonal component $\left(\sigma_{x y}\right)$, which is associated with the deviation of the tilting axis of the Dirac cone from the $k_{x}$ or $k_{y}$ axis, results in the principal axis with clockwise or anticlockwise rotation depending on the sign of $\sigma_{x y}$.

Finally, we compare our result with that of the experiment. The temperature dependence of resistance (corresponding to the inverse of the conductivity) under hydrostatic pressure shows a nearly constant behavior at high temperatures and a minimum at low temperatures, whereas the minimum is invisible under uniaxial pressure. ${ }^{2)}$ Our results show the broad maximum for $\sigma_{y}$ and monotonic variation for $\sigma_{x}$. Although we obtain the qualitative coincidence, the details of the correspondence between them, e.g., the direction of measurement, are needed for the quantitative comparison. It also remains a future problem to clarify if the conductivity under pressure suggests the presence of site potentials. The validity of our present calculation may be examined by the measurement of the deviation angle of the principal axis, i.e., clockwise (anticlockwise).

\section{Acknowledgements}

We thank R. Kato for useful discussions on the effect of the $\mathrm{e}-\mathrm{p}$ interaction. This work was supported by Grantsin-Aid for Scientific Research from the Japan Society for the Promotion of Science (Grant No. JP18H01162), and by JST-Mirai Program (Grant No. JPMJMI19A1).

\section{Appendix A: Matrix element of $\mathbf{H}$}

The quantity $h_{i, j}$, which denotes the matrix element of $H$ and is the same as that of Ref.8, is given as

$$
\begin{aligned}
h_{12}(\boldsymbol{k}) & =a_{3}+a_{2} Y, \\
h_{13}(\boldsymbol{k}) & =b_{3}+b_{2} X, \\
h_{14}(\boldsymbol{k}) & =b_{4} Y+b_{1} X Y, \\
h_{23}(\boldsymbol{k}) & =b_{2}+b_{3} X, \\
h_{24}(\boldsymbol{k}) & =b_{1}+b_{4} X, \\
h_{34}(\boldsymbol{k}) & =2 a_{1}, \\
h_{11}(\boldsymbol{k}) & =t_{22}(\boldsymbol{k})=a_{1 d}(Y+\bar{Y}), \\
h_{33}(\boldsymbol{k}) & =a_{3 d}(Y+\bar{Y})+\tilde{V}_{\mathrm{B}}, \\
h_{44}(\boldsymbol{k}) & =a_{4 d}(Y+\bar{Y})+\tilde{V}_{\mathrm{C}},
\end{aligned}
$$

and $h_{i j}(\boldsymbol{k})=h_{j i}^{*}(\boldsymbol{k})$, where $X=\exp [i k x]=\bar{X}^{*}$ and $Y=\exp [i k y]=\bar{Y}^{*}$,

The matrix elements of $H_{1}$ are $\tilde{V}_{B}$ for $\alpha=\beta=3, \tilde{V}_{C}$ for $\alpha=\beta=4$, and zero otherwise. The site potentials $\tilde{V}_{\mathrm{B}}$ and $\tilde{V}_{\mathrm{C}}$ in $H_{1}$ are given by the mean-field of short-range repulsive interactions, ${ }^{8)}$

$$
\begin{aligned}
\tilde{V}_{\mathrm{B}} & =\left(n_{B}-n_{A}\right) U / 2 \\
& +2 V_{a}\left(n_{C}-n_{A}\right)+2 V_{b}\left(2 n_{A}-n_{B}-n_{C}\right),
\end{aligned}
$$

$\tilde{V}_{\mathrm{C}}=\left(n_{C}-n_{A}\right) U / 2$ 


$$
+2 V_{a}\left(n_{B}-n_{A}\right)+2 V_{b}\left(2 n_{A}-n_{B}-n_{C}\right),
$$

where $U$ is the on-site repulsive interaction and $V_{a}\left(V_{b}\right)$ denotes the nearest neighbor interaction along the $y(x)$ axis. $n_{\alpha}$ denotes a local density corresponding to an electron number per unit cell at the $\alpha$ site and is determined self-consistently. In the present case, the site potentials given by Eqs. (A.2a) and (A.2b) are estimated as $\tilde{V}_{B}=0.0511$ and $\tilde{V}_{C}=0.0032$, respectively, for $U=$ $0.4, V_{a}=0.17$, and $V_{b}=0.05$, where $n_{A}=n_{A^{\prime}}=1.46, n_{B}$ $=1.37$, and $n_{C}=1.71$, and $\mu=0.172$ at $T=0$.

\section{Appendix B: Damping by phonon scattering}

The damping of electrons of the $\gamma$ band, which is defined by $\Gamma_{\gamma}$, is obtained from the electron Green function $^{35)}$ expressed as

$$
\begin{aligned}
G_{\gamma}\left(\boldsymbol{k}, i \omega_{n}\right)^{-1} & =i \omega_{n}-E_{\gamma, \boldsymbol{k}}+\mu+i \Gamma_{\gamma}, \\
\Gamma_{\gamma} & =\Gamma+\Gamma_{\mathrm{ph}}^{\gamma},
\end{aligned}
$$

where $\Gamma_{\mathrm{ph}}^{\gamma}=-\operatorname{Im} \Sigma_{\gamma}\left(\boldsymbol{k}, E_{\gamma, \boldsymbol{k}}-\mu\right)$ with $\Sigma_{\gamma}\left(\boldsymbol{k}, E_{\gamma, \boldsymbol{k}}-\mu\right)$ being a self-energy given by the e-p interaction. The real part of the self-energy can be neglected for doping at low concentrations. ${ }^{27)}$ The quantity $\Gamma$ comes from another self-energy by the impurity scattering. Note that $\Gamma_{\mathrm{ph}}^{\gamma}$ does not depend on $\Gamma$, and that the ratio $\Gamma_{\mathrm{ph}}^{\gamma} / \Gamma$ is crucial to the determination of the $T$ dependence of the conductivity. The quantity $\Sigma_{\gamma}(\boldsymbol{k}, \omega)=\Sigma_{\gamma}\left(\boldsymbol{k}, i \omega_{n}\right)$ with $i \omega_{n} \rightarrow \omega+0$ is estimated $\mathrm{as}^{35)}$

$$
\begin{aligned}
& \Sigma_{\gamma}\left(\boldsymbol{k}, i \omega_{n}\right)=T \sum_{m} \sum_{\boldsymbol{q}}\left|\alpha_{q}\right|^{2} \\
& \times \frac{1}{i \omega_{n+m}-\xi_{\gamma, \boldsymbol{k}+\boldsymbol{q}}} \times \frac{2 \omega_{\boldsymbol{q}}}{\omega_{m}^{2}+\omega_{\boldsymbol{q}}^{2}},
\end{aligned}
$$

which is a product of electron and phonon Green functions. $\omega_{n}=(2 n+1) \pi T, \omega_{m}=2 \pi m T$ with $n$ and $m$ being integers. $\xi_{\gamma, \boldsymbol{k}}=E_{\gamma, \boldsymbol{k}}-\mu$. Applying the previous result, ${ }^{27)}$ we obtain Eqs. (14a) and (14b).

1) K. S. Novoselov, A. K. Geim, S. V. Morozov, D. Jiang, M. I. Katsnelson, I. V. Grigorieva, S. V. Dubonos, and A. A. Firsov, Nature 438, 197 (2005).

2) K. Kajita, Y. Nishio, N. Tajima, Y. Suzumura, and A. Kobayashi, J. Phys. Soc. Jpn. 83, 072002 (2014).

3) T. Mori, A. Kobayashi, Y. Sasaki, H. Kobayashi, G. Saito, and H. Inokuchi, Chem. Lett. 13, 957 (1984).

4) A. Kobayashi, S. Katayama, K. Noguchi, and Y. Suzumura, J. Phys. Soc. Jpn. 73, 3135 (2004).

5) S. Katayama, A. Kobayashi, and Y. Suzumura, J. Phys. Soc.
Jpn. 75, 054705 (2006).

6) R. Kondo, S. Kagoshima, and J. Harada, Rev. Sci. Instrum. 76, 093902 (2005).

7) H. Kino and T. Miyazaki, J. Phys. Soc. Jpn. 75, 034704 (2006).

8) S. Katayama, A. Kobayashi, and Y. Suzumura, Eur. Phys. J. B 67, 139 (2009).

9) M. Inokuchi, H. Tajima, A. Kobayashi, H. Kuroda, R. Kato, T. Naito, and H. Kobayashi, Synth. Met. 56, 2495 (1993).

10) M. Inokuchi, H. Tajima, A. Kobayashi, T. Ohta, H. Kuroda, R. Kato, T. Naito, and H. Kobayashi, Bull. Chem. Soc. Jpn. 68, 547 (1995)

11) R. Kondo, S. Kagoshima, N. Tajima, and R. Kato, J. Phys. Soc. Jpn. 78, 114714 (2009).

12) T. Morinari and Y. Suzumura, J. Phys. Soc. Jpn. 83, 094701 (2014).

13) T. Naito, R. Doi, and Y. Suzumura, J. Phys. Soc. Jpn. 89, 023701 (2020).

14) K. Kajita, T. Ojiro, H. Fujii, Y. Nishio, H. Kobayashi, A. Kobayashi, and R. Kato, J. Phys. Soc. Jpn. 61, 23 (1992).

15) N. Tajima, M. Tamura, Y. Nishio, K. Kajita, and Y. Iye, J. Phys. Soc. Jpn. 69, 543 (2000).

16) N. Tajima, A. Ebina-Tajima, M. Tamura, Y. Nishio, and K. Kajita, J. Phys. Soc. Jpn. 71, 1832 (2002).

17) N. Tajima, S. Sugawara, M. Tamura, R. Kato, Y. Nishio, and K. Kajita, EPL 80, 47002 (2007).

18) D. Liu, K. Ishikawa, R. Takehara, K. Miyagawa, M. Tanuma, and K. Kanoda, Phys. Rev. Lett. 116, 226401 (2016).

19) R. Kato, H. Cui, T. Tsumuraya, T. Miyazaki, and Y. Suzumura, J. Am. Chem. Soc. 139, 1770 (2017).

20) R. Kato and Y. Suzumura, J. Phys. Soc. Jpn. 86, 064705 (2017).

21) Y. Suzumura, H. B. Cui, and R. Kato, J. Phys. Soc. Jpn. 87, 084702 (2018).

22) B. Zhou, S. Ishibashi, T. Ishii, T. Sekine, R. Takehara, K. Miyagawa, K. Kanoda, E. Nishibori, and A. Kobayashi, Chem. Commun. 55, 3327 (2019).

23) R. Kato and Y. Suzumura, J. Phys. Soc. Jpn. 89, 044713 (2020).

24) N. H. Shon and T. Ando, J. Phys. Soc. Jpn. 67, 2421 (1998).

25) Y. Suzumura, I. Proskurin, and M. Ogata J. Phys. Soc. Jpn. 83, 023701 (2014).

26) N. M. R. Peres, F. Guinea, and A. H. Castro Neto, Phys. Rev. B 83,125411 (2006).

27) Y. Suzumura and M. Ogata, Phys. Rev. B 98, 161205 (2018).

28) A. Kobayashi, S. Katayama, Y. Suzumura, and H. Fukuyama, J. Phys. Soc. Jpn. 76, 034711 (2007).

29) M. O. Goerbig, J.-N. Fuchs, G. Montambaux, and F. Piéchon, Phys. Rev. B 78, 045415 (2008).

30) A. Kobayashi, Y. Suzumura, and H. Fukuyama, J. Phys. Soc. Jpn. 77, 064718 (2008)

31) H. Fröhlich, Proc. Phys. Soc. A 223, 296 (1954).

32) T. Holstein, Ann. Phys. 29, 410 (1964).

33) S. Katayama, A. Kobayashi, and Y. Suzumura, J. Phys. Soc. Jpn. 75, 023708 (2006).

34) For example, see Eq. (6.9) in R. Kubo, J. Phys. Soc. Jpn. 12, 570 (1957)

35) A. A. Abrikosov, L. P. Gorkov, and I. E. Dzyaloshinskii, Methods of Quantum Field Theory in Statistical Physics (Prentice-Hall, Englewood Cliffs, NJ, 1963). 